\title{
Case Report of Urinary Schistosomiasis in a Returned Traveler in Korea
}

\author{
Yangkyu Lee', Hyun Beom Song ${ }^{2}$, Bong-Kwang Jung ${ }^{2,3}$, Gheeyoung Choe', Min-Ho Choi',** \\ ${ }^{1}$ Department of Pathology, Seoul National University Bundang Hospital, Seoul National University College of Medicine, Seongnam 13620, Korea; \\ ${ }^{2}$ Department of Tropical Medicine and Parasitology and Institute of Endemic Diseases, Seoul National University College of Medicine, Seoul 03080, \\ Korea; ${ }^{3}$ nstitute of Parasitic Diseases, Korea Association of Health Promotion, Seoul 07649, Korea
}

\begin{abstract}
A 23-year-old Korean woman with a residence history in Kenya and Malawi for about 2 years presented with gross hematuria for 1 month. Blood tests were within normal range except eosinophilia. Asymmetrically diffuse wall thickening and calcification were observed at the urinary bladder on CT. Multiple erythematous nodular lesions were observed in the cystoscopy and transurethral resection was done. Numerous eggs of Schistosoma haematobium with granulomatous inflammation were observed in the submucosal layer of the bladder. The patient was diagnosed with schistosomiasis-related cystitis and treated with praziquantel ( $40 \mathrm{mg} / \mathrm{kg} / \mathrm{day})$ twice before and after transurethral resection. This case suggests that $S$. haematobium infection should be considered as a cause of hematuria in Korea when the patient had a history of traveling endemic areas of schistosomiasis.
\end{abstract}

Key words: Schistosoma haematobium, urinary bladder, hematuria, cystitis

\section{INTRODUCTION}

Schistosomiasis is one of the neglected tropical diseases (NTDs) caused by the genus Schistosoma, which affects over 200 million people worldwide [1]. There are 6 species of schistosomes infecting human beings: S. haematobium, S. mansoni, S. japonicum, S. mekongi, S. intercalatum and S. guineensis [1]. Among them, S. haematobium induces urogenital schistosomiasis, while the others are the cause of intestinal schistosomiasis. People are infected by contact with water infested with cercariae of schistosome during recreational, occupational, agricultural, and daily activities [2]. It is estimated that at least $91.4 \%$ of infected persons requiring treatment live in Africa, and school-age children are the most risk group because they tend to spend time swimming, bathing or fishing in water $[2,3]$.

Schistosomiasis is not endemic in Korea and therefore, only the imported cases have been reported in travelers to endemic areas of schistosomiasis. In this report, we presented a case of

\footnotetext{
- Received 2 July 2019, revised 26 November 2019, accepted 4 December 2019.

*Corresponding author (mhchoi@snu.ac.kr)

(C) 2020, Korean Society for Parasitology and Tropical Medicine

This is an Open Access article distributed under the terms of the Creative Commons Attribution Non-Commercial License (https://creativecommons.org/licenses/by-nc/4.0) which permits unrestricted non-commercial use, distribution, and reproduction in any medium, provided the original work is properly cited.
}

S. haematobium infection in a Korean woman with a residence history in Kenya and Malawi in South Africa, which has been diagnosed with schistosomiasis-related cystitis.

\section{CASE RECORD}

A 23-year-old Korean woman presented with painless gross hematuria for one month in July 2016. The first episode occurred 3 years ago in 2013, but the subsequent histories related to hematuria were not clear. In 2014, she was treated with antibiotics in suspicion of acute cystitis for 1 week because of chilling sense and shivering symptoms without voiding symptoms. The patient currently lives in the United States, but she spent about 2 years from 2011 to 2013 in Kenya and Malawi as a teenager.

Hematuria had been continued at the time of admission. Urinalysis showed hematuria, proteinuria, and pyuria with a positive nitrite result. In urine cytology, abnormal findings were not identified. Blood tests revealed eosinophilia $(1,129 /$ $\mu \mathrm{l}, 19.5 \%$ ) without anemia (hemoglobin $13.2 \mathrm{~g} / \mathrm{dl}$ ). Asymmetrically diffuse mucosal wall thickening and calcification were observed at the urinary bladder on pelvic CT (Fig. 1). Multiple erythematous nodular lesions and hemorrhage at the urinary bladder mucosa were observed in the cystoscopy and transurethral resection was performed for the histopathologic 


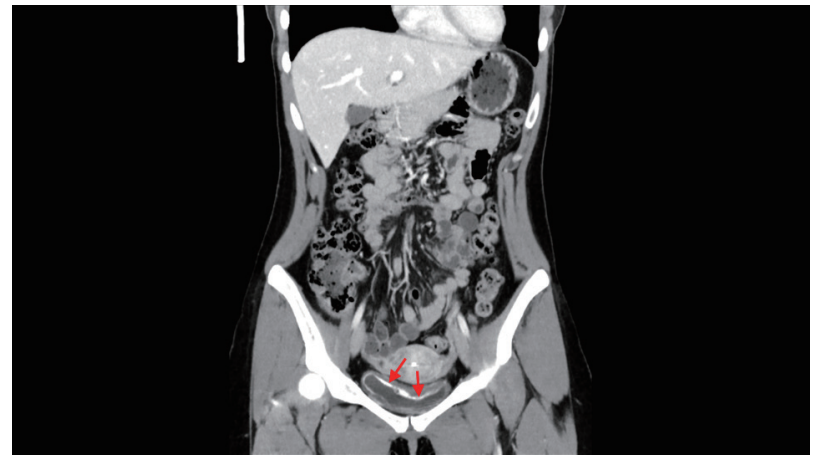

Fig. 1. Asymmetrical thickening of the mucosal wall with calcification (arrows) at the urinary bladder on pelvic CT.
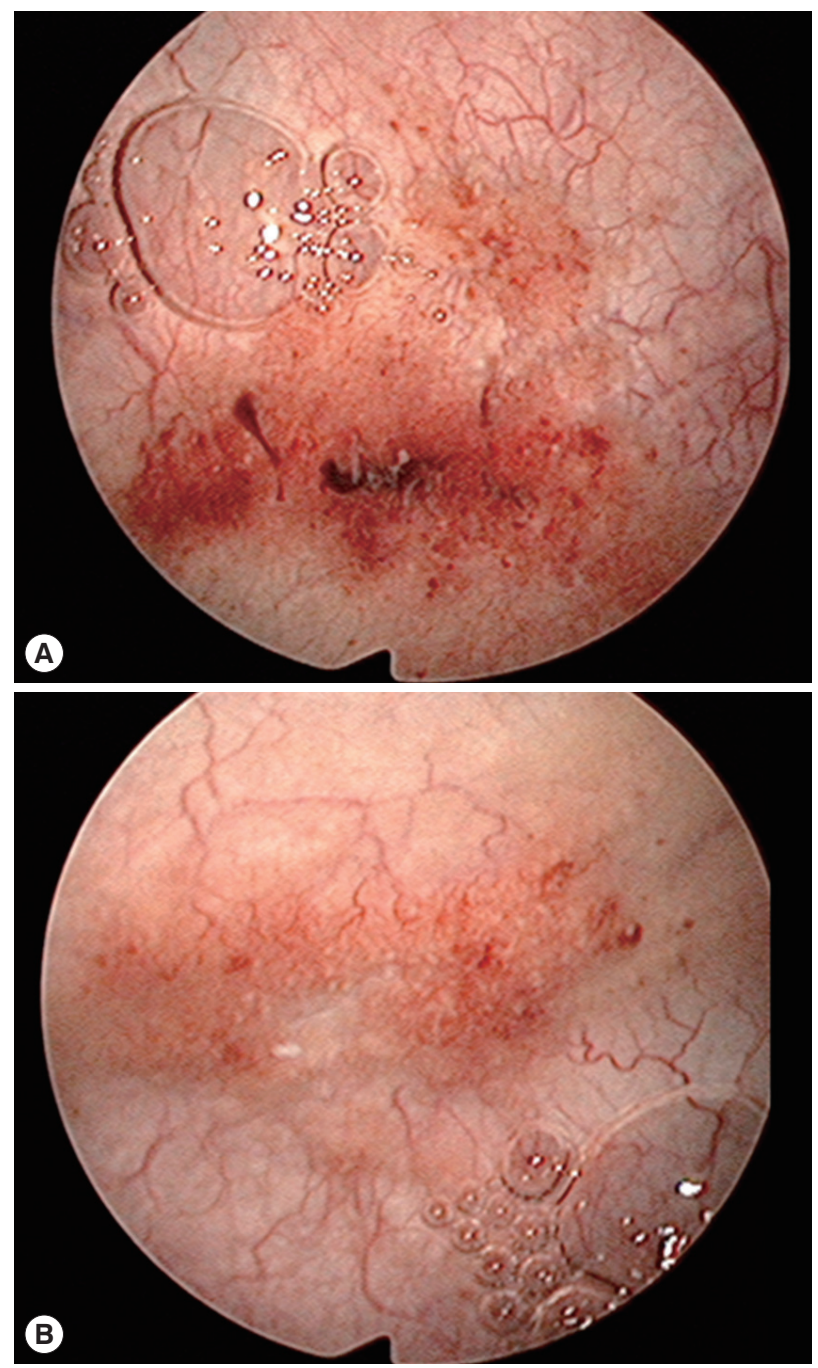

Fig. 2. "Sandy patches" appearance with erythema and hemorrhage in the urinary bladder on cystoscopy (A, B).

examination for the lesions (Fig. 2).

In histopathologic findings, numerous calcified or non-cal-
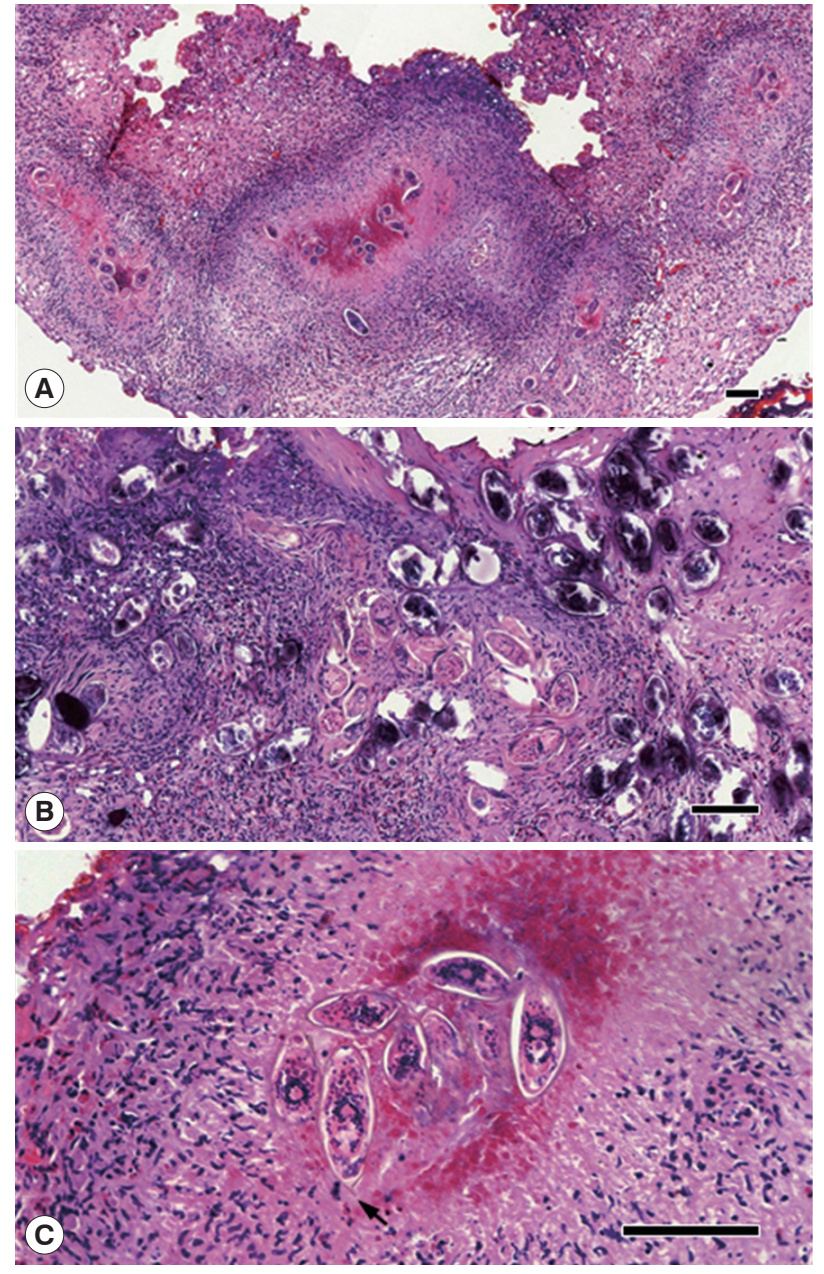

Fig. 3. Histopathologic findings of the lesion. (A) Chronic granulomatous inflammation around eggs of Schistosoma haematobium. Bar $=100 \mu \mathrm{m}(\times 100)$. (B) Numerous eggs with dystrophic calcification in the submucosal layer of the bladder. Bar $=100 \mu \mathrm{m}$ (×200). (C) Eggs of S. haematobium in ellipsoidal shape with a single terminal spine (an arrow). Bar $=100 \mu \mathrm{m}(\times 400)$.

cified parasite eggs were observed in the submucosal layer of the urinary bladder (Fig. 3). Many granulomas were formed around parasite eggs with foreign-body type multinucleated giant cells. Diffuse fibrosis and dystrophic calcification were observed in addition to eosinophilic infiltration and submucosal hemorrhage on hematoxylin and eosin (H\&E) stained slides. Eggs were approximately above $100 \mu \mathrm{m}$ in size and were embryonated with elongated or ellipsoid shape and a terminal spine (Fig. 3). However, an operculum was not identified.

The patient was diagnosed with schistosomiasis-related cystitis considering the symptoms, histopathologic findings, and the past history of living in endemic areas of schistosomiasis although the route of infection is not definite. She was treated 
with praziquantel $(40 \mathrm{mg} / \mathrm{kg} /$ day) twice before and after transurethral resection, and did not complain of any urinary symptoms after the treatment. At the last follow-up 8 months after the treatment, she remained free of urinary symptoms and the eggs were not detected in the urine. However, polymerase chain reaction (PCR) analysis of the urine was positive for $S$. haematobium and the patient serum evaluated by enzymelinked immunosorbent assay (ELISA) was positive for specific IgG antibodies against soluble egg antigens of $S$. haematobium. As the positive PCR results in the urine and positive ELISA result in the blood were considered to be a result of the past infection, no additional treatment was given.

\section{DISCUSSION}

Schistosomiasis is prevalent in tropical or subtropical areas in Africa, the Middle East, Southeast Asia, the Caribbean, and South America, but it occurs mostly in sub-Saharan Africa [2]. It is not endemic in Korea as the snail intermediate hosts are not available, and therefore, all the infected cases were imported from endemic countries. The first description of schistosomiasis in Korea is renal schistosomiasis that has been identified from a prisoner of wars during the Korean War, and it was assumed that he might have moved from China or Japan to Korea after the independence of Korea from Japanese ruling [4]. Since then, a total of 13 schistosomiasis has been reported in the literature until now: 6 cases by $S$. haematobium, 4 by $S$. mansoni, one by $S$. japonicum, and 2 unidentified cases $[5,6]$. Among them, 10 cases were Korean with a residence history in the Middle East including North Yemen and Saudi Arabia, and 3 cases were reported from one Ghanaian and 2 Philippine patients, respectively [5-7].

The present case is the 15th cases reported in the literature so far and it is suspected that the patient was exposed to water infested with schistosome cercariae during her residence in Kenya and Malawi, where 2 major forms of schistosomiasis are prevalent. Both urogenital and intestinal schistosomiasis are prevalent in Lake Malawi, one of the largest lakes in Central Africa, where co-infection occurs in infected schoolchildren [8]. In Scotland, the majority of new cases of schistosomiasis between 2005 and 2009 were identified from travelers returning from Malawi [9]. Cross-sectional surveys in over 20,000 schoolchildren in 200 schools across Kenya performed in 2012 showed the overall prevalence of $S$. mansoni as $2.1 \%$ and the prevalence and intensity were highest in the Western Province, while the prevalence of $S$. haematobium was $14.8 \%$ in the Coast Province [10]. Despite preventive measures such as mass drug administration in primary schools, schistosomiasis still remains a major public health problem in Kenya, especially along the shores of Lake Victoria [11].

Morbidity caused by schistosome infections is induced by host reaction to eggs of Schistosoma rather than by adult worm itself [1]. Hematuria is the classic symptom of urogenital schistosomiasis and often is presented with urinary frequency, burning micturition, and suprapubic pain or discomfort [1]. Fibrosis of the bladder and the ureter are sometimes observed in advanced cases [2]. In men, urogenital schistosomiasis can induce pathology of the seminal vesicles, prostate, and infertility may occur if the infection lasts long without treatment $[1,2]$. In women, urogenital schistosomiasis strongly affects women's health by inducing inflammatory lesions of the reproductive organs and can increase the risk of abortion and transmission of HIV infection [1]. It is well-known that $S$. haematobium infection is strongly associated with squamous cell carcinoma of the bladder [1].

The microscopic observation of eggs in urine or stool is the gold standard for the diagnosis of schistosomiasis. The egg's size, shape, and a typical spine can be diagnostic clues [12]. Serological assays can detect antibodies against schistosomal antigens in the blood, and are useful especially for the diagnosis for symptomatic travelers [1]. However, it is difficult to discriminate between the active infection and past exposure in people in endemic areas [1], and cross-reaction with other parasites can occur [13]. PCR techniques have also been introduced for diagnosis [14] and DNA detection for serum or urine was developed and being evaluated $[15,16]$.

Cystoscopy and endoscopy for visualization of the lesion are also used in hospital settings. 'Sandy patches' appearance, mucosal nodularity, is one of the cystoscopic findings for urinary schistosomiasis [17]. If the submucosa is involved, fibrosis or calcification affects the contraction of the urinary bladder, resulting in hypertonic or atonic bladder [17]. Radiologic techniques allow visualization of pathologic lesions by $S$. haematobium infection, and calcification of the ureter and bladder wall due to the presence of calcified eggs in the submucosa is the most common radiologic finding in endemic areas [18].

Praziquantel as a single dose ( $40 \mathrm{mg} / \mathrm{kg} /$ day) is effective for urogenital schistosomiasis and the therapeutic effect can be determined by the decreased number of eggs excreted in urine [1]. In the present case, praziquantel treatment achieved para- 
site-free status without parasite eggs in the urine, but ELISA and PCR showed positive results. As IgG antibodies against soluble egg antigen remained above the cut-off levels up to 24 months in some cured patients [19], the positive ELISA result does not necessarily indicate a current infection. In addition, a substantial proportion of patients was positive for PCR even after praziquantel treatment, which was considered as a result of re-infection, partial treatment failure, post-treatment maturation of juvenile worms or lingering ova and parasite products in the tissue [20]. In this case, the patient was not exposed to the source of infection after the treatment and remained free of symptoms and no eggs in the urine 8 months after the treatment. Therefore, it is suggested that the positivity for PCR indicates the persistent release of $S$. haematobium egg particles from the bladder wall granuloma. This patient appears to represent an irreversible and chronic status in spite of the treatment with praziquantel and further management will be necessary for the future.

S. haematobium infection should be considered as a cause of urinary symptoms in people who have a travel or residence history in endemic areas. It is important to take appropriate action and treat it promptly when there is a possibility of exposure to the contaminated water.

\section{ACKNOWLEDGMENTS}

This study was supported by Seoul National University Hospital.

\section{CONFLICT OF INTEREST}

The authors have no potential conflicts of interest to disclose.

\section{REFERENCES}

1. Colley DG, Bustinduy AL, Secor WE, King CH. Human schistosomiasis. Lancet 2014; 383: 2253-2264.

2. Schistosomiasis [Internet]; [cited 2019 Mar 14]. Available from: https://www.who.int/news-room/fact-sheets/detail/schistosomiasis.

3. Steinmann P, Keiser J, Bos R, Tanner M, Utzinger J. Schistosomiasis and water resources development: systematic review, metaanalysis, and estimates of people at risk. Lancet Infect Dis 2006; 6: 411-425.

4. Huh S. Parasitic diseases as the cause of death of prisoners of war during the Korean War (1950-1953). Korean J Parasitol 2014; 52: 335-337.

5. Joo KH. International travel and imported parasitic diseases. Hanyang Med Rev 2010; 30: 156-175 (in Korean).

6. Lee HJ, Sung WS. Calcification of the urinary bladder and ureter in schistosomiasis. Kidney Res Clin Pract 2018; 37: 304-305.

7. Hong CK, Yang JM, Kang BK, Kim JD, Kim YC, Chang UI, Yoo JY. A case of combined hepatocellular-cholangiocarcinoma with underlying schistosomiasis. Korean J Intern Med 2007; 22: 283286.

8. Alharbi MH, Condemine C, Christiansen R, LaCourse EJ, Makaula P, Stanton MC, Juziwelo L, Kayuni S, Stothard JR. Biomphalaria pfeifferi snails and intestinal schistosomiasis, Lake Malawi, Africa, 2017-2018. Emerg Infect Dis 2019; 25: 613-615.

9. Blach O, Rai B, Oates K, Franklin G, Bramwell S. An outbreak of schistosomiasis in travellers returning from endemic areas: the importance of rigorous tracing in peer groups exposed to risk of infection. J Public Health (Oxf) 2012; 34: 32-36.

10. Mwandawiro CS, Nikolay B, Kihara JH, Ozier O, Mukoko DA, Mwanje MT, Hakobyan A, Pullan RL, Brooker SJ, Njenga SM. Monitoring and evaluating the impact of national school-based deworming in Kenya: study design and baseline results. Parasit Vectors 2013; 6: 198.

11. Takeuchi R, Njenga SM, Ichinose Y, Kaneko S, Estrada CA, Kobayashi J. Is there a gap between health education content and practice toward schistosomiasis prevention among schoolchildren along the shores of Lake Victoria in Kenya? PLoS Negl Trop Dis 2019; 13: e0007572.

12. Feldmeier H, Poggensee G. Diagnostic techniques in schistosomiasis control. A review. Acta Trop 1993; 52: 205-220.

13. Salah F, El Bassiouny A, Rabia I, Demerdash Z, Roshdy M, Shaker Z. Human schistosomiasis haematobium: effective diagnosis of active infection using a pair of monoclonal antibodies against soluble egg antigen. Parasitol Res 2006; 99: 528-533.

14. Sady H, Al-Mekhlafi HM, Ngui R, Atroosh WM, Al-Delaimy AK, Nasr NA, Dawaki S, Abdulsalam AM, Ithoi I, Lim YA, Chua KH, Surin J. Detection of Schistosoma mansoni and Schistosoma haematobium by real-time PCR with high resolution melting analysis. Int J Mol Sci 2015; 16: 16085-16103.

15. Ibironke O, Koukounari A, Asaolu S, Moustaki I, Shiff C. Validation of a new test for Schistosoma haematobium based on detection of Dra1 DNA fragments in urine: evaluation through latent class analysis. PLoS Negl Trop Dis 2012; 6: e1464.

16. Wichmann D, Poppert S, Von Thien H, Clerinx J, Dieckmann S, Jensenius M, Parola P, Richter J, Schunk M, Stich A, Zanger P, Burchard GD, Tannich E. Prospective European-wide multicentre study on a blood based real-time PCR for the diagnosis of acute schistosomiasis. BMC Infect Dis 2013; 13: 55.

17. Barsoum RS. Urinary schistosomiasis: review. J Adv Res 2013; 4: 453-459.

18. Shebel HM, Elsayes KM, Abou El Atta HM, Elguindy YM, El-Diasty TA. Genitourinary schistosomiasis: Life cycle and radiologicpathologic findings. Radiographics 2012; 32: 1031-1046. 
19. Rabello AL, Garcia MM, Pinto da Silva RA, Rocha RS, Katz N. Humoral immune responses in patients with acute Schistosoma mansoni infection who were followed up for two years after treatment. Clin Infect Dis 1997; 24: 304-308.

20. Downs JA, Kabangila R, Verweij JJ, Jaka H, Peck RN, Kalluvya SE,
Changalucha JM, Johnson WD, van Lieshout L, Fitzgerald DW. Detectable urogenital schistosome DNA and cervical abnormalities 6 months after single-dose praziquantel in women with Schistosoma haematobium infection. Trop Med Int Health 2013; 18: 1090-1096. 
